\title{
Structure of a eukaryotic thiaminase I
}

\author{
Cheryl A. Kreinbring ${ }^{a}$, Stephen P. Remillard ${ }^{b, 1}$, Paul Hubbard ${ }^{a, 2}$, Heather R. Brodkin ${ }^{a}$, Finian J. Leeper ${ }^{c}$, Dan Hawksleyc, \\ Elaine Y. Lai ${ }^{\mathrm{b}}$, Chandler Fulton ${ }^{\mathrm{b}}$, Gregory A. Petsko ${ }^{\mathrm{a}, 3}$, and Dagmar Ringe ${ }^{\mathrm{a}, 3}$
}

${ }^{a}$ Department of Biochemistry and Chemistry, Rosenstiel Basic Medical Sciences Research Center, and ${ }^{\mathrm{b}}$ Department of Biology, Brandeis University, Waltham, MA 02454-9110; and 'Department of Chemistry, University of Cambridge, Cambridge CB2 1EW, United Kingdom

Contributed by Gregory A. Petsko, August 30, 2013 (sent for review December 20, 2012)

\begin{abstract}
Thiaminases, enzymes that cleave vitamin B1, are sporadically distributed among prokaryotes and eukaryotes. Thiaminase I enzymes catalyze the elimination of the thiazole ring moiety from thiamin through substitution of the methylene group with a nitrogenous base or sulfhydryl compound. In eukaryotic organisms, these enzymes are reported to have much higher molecular weights than their bacterial counterparts. A thiaminase I of the singlecelled amoeboflagellate Naegleria gruberi is the only eukaryotic thiaminase I to have been cloned, sequenced, and expressed. Here, we present the crystal structure of $\boldsymbol{N}$. gruberi thiaminase I to a resolution of $2.8 \AA$, solved by isomorphous replacement and pseudotwo-wavelength multiwavelength anomalous diffraction and refined to an $R$ factor of $0.231\left(R_{\text {free }}, 0.265\right)$. This structure was used to solve the structure of the enzyme in complex with 3-deazathiamin, a noncleavable thiamin analog and enzyme inhibitor ( $2.7 \AA$ \& $R, 0.233$; $\left.R_{\text {free }}, 0.267\right)$. These structures define the mode of thiamin binding to this class of thiaminases and indicate the involvement of Asp272 as the catalytic base. This enzyme is able to use thiamin as a substrate and is active with amines such as aniline and veratrylamine as well as sulfhydryl compounds such as L-cysteine and $\beta$-mercaptoethanol as cosubstrates. Despite significant differences in polypeptide sequence and length, we have shown that the $N$. gruberi thiaminase $\mathrm{I}$ is homologous in structure and activity to a previously characterized bacterial thiaminase $\mathrm{I}$.
\end{abstract}

X-ray crystallography | thiamin degradation

$\mathbf{T}^{\mathrm{t}}$ he nonpathogenic unicellular protozoan Naegleria gruberi is a ubiquitous eukaryote, found in aerobic and microaerobic environments including freshwater, freshwater silt, and moist soils worldwide (1-4). It exists predominantly as an amoeba but is able to undergo a rapid phenotypic change into a streamlined swimming flagellate when subjected to a nutrient-poor environment (3). Naegleria is also able to form resting cysts, which are then capable of excysting back to amoebae (2).

Extracts of $N$. gruberi have been shown to possess a proteinaceous agent that is "cytopathogenic" to vertebrate cells (5). Fulton and Lai show that these extracts of $N$. gruberi amoebae cause generations-delayed apoptotic death of both proliferating and quiescent vertebrate cells in culture (6). The apoptosisinducing agent was isolated based on activity, and characterized and identified as a thiaminase I, an enzyme that degrades thiamin (vitamin B1) and thiamin diphosphate (TPP), the biologically active form of thiamin (7). They showed that $N$. gruberi thiaminase I induces apoptosis via its enzymatic activity in that an enzymatically inactive mutant lost its ability to cause cell death (7). Active thiaminase I depletes extracellular thiamin, which leads to subsequent depletion of intracellular TPP, an essential coenzyme for many enzymes involved in carbohydrate and energy metabolism, and in turn triggers apoptosis by an asyet-to-be-characterized mechanism. It has long been known that thiamin deficiency in animals can cause neurological and cardiac symptoms that ultimately lead to death of the animal. In humans, beriberi and Wernicke-Korsakoff syndrome are associated with chronic thiamin deficiency (8). Fulton and Lai have shown that the $N$. gruberi thiaminase I is also capable of killing cells resistant to commonly used chemotherapeutic agents and suggest that thiamin depletion-induced cell death may be a suitable candidate for use in tissue-targeted cancer therapies (6).
Thiaminases catalyze the cleavage of biologically active thiamin into its pyrimidine and thiazole ring moieties $(9,10)$. These enzymes can be grouped into two classes defined by the nucleophile used in the mechanism by which the cleavage is accomplished. The thiaminase II (EC 3.5.99.2) class of enzymes exclusively uses water to accelerate the hydrolysis of thiamin into 2-methyl-4-amino-5-hydroxymethylpyrimidine (HMP) and 4-methyl-5-(2-hydroxyethyl)thiazole and is found only in bacteria, fungi, and yeast (11). The thiaminase I (EC 2.5.1.2) class of enzymes uses a variety of aromatic and heterocyclic amines and sulfhydryl compounds as substituting bases in a nucleophilic displacement reaction on the methylene group of the pyrimidine moiety $(10,12-15)$. Thiaminase $I$ is found in specific species of microorganisms such as Bacillus and Clostridium as well as multicellular organisms including certain ferns, insects, shellfish, and freshwater and ocean fish $(10,14,16-26)$. Although distributed throughout the kingdoms, the phylogenetic distribution of thiaminase I, unlike most other enzymes, appears to follow no easily discernable evolutionary pattern. A physiological role has yet to be assigned for thiaminase I (27), and several studies report that thiaminase I enzymes from eukaryotic organisms are produced as larger holoenzymes, $55-200 \mathrm{kDa}$, some of which are active as smaller fragments $(17,21-23,25,26)$. The presence of this enzyme in the diet of animals can have several deleterious effects and can even be fatal. It is responsible for thiamin deficiency and early mortality syndrome in Great Lakes salmonines, polioencephalomalacia in sheep, and cerebrocortical

\section{Significance}

Thiaminases, enzymes that cleave vitamin B1 into its pyrimidine and thiazole ring moieties, are sporadically distributed among prokaryotes and eukaryotes. Thiaminase I enzymes accomplish this reaction through substitution of the thiazole ring with a nitrogenous base or sulfhydryl compound. A thiaminase I of the single-celled amoeboflagellate Naegleria gruberi is the first eukaryotic thiaminase I to have been examined structurally. The crystal structures in both apo form and bound to 3-deazathiamin, a noncleavable thiamin analog and inhibitor of the enzyme, define the mode of thiamin binding to this class of thiaminases and indicate the residues important for catalysis. Comparison with thiaminase II argues for convergent evolution between these two enzymes.

Author contributions: C.A.K., S.P.R., and H.R.B. designed research; C.A.K. and S.P.R. performed research; S.P.R., F.J.L., D.H., E.Y.L., and C.F. contributed new reagents/analytic tools; C.A.K., P.H., and H.R.B. analyzed data; and C.A.K., P.H., H.R.B., G.A.P., and D.R. wrote the paper.

The authors declare no conflict of interest.

Data deposition: The atomic coordinates and structure factors have been deposited in the Research Collaboratory for Structural Bioinformatics Protein Data Bank, www.rcsb.org [ID codes 4HCW (apo Ng-thiaminase) and 4HCY (Ng-thiaminase bound to 3-deazathiamin)]. 1Present address: Department of Medical Oncology, Ludwig Center for Cancer Research Lab/Medical Oncology D836, Dana-Farber Cancer Institute, Boston, MA 02215.

${ }^{2}$ Present address: BioFocus, Chesterford Research Park, Saffron Walden CB10 1XL, United Kingdom.

${ }^{3}$ To whom correspondence may be addressed. E-mail: petsko@brandeis.edu or ringe@ brandeis.edu.

This article contains supporting information online at www.pnas.org/lookup/suppl/doi:10. 1073/pnas.1315882110/-/DCSupplemental. 


\begin{tabular}{|c|c|c|c|c|}
\hline \multicolumn{5}{|c|}{ Full-length Naegleria protein } \\
\hline Cys & Glu Asp & Glu & Glu & \\
\hline 118 & 241272 & 513 & 761 & \\
\hline & & & & \\
\hline
\end{tabular}

Bacillus thiaminolyticus thiaminase-I
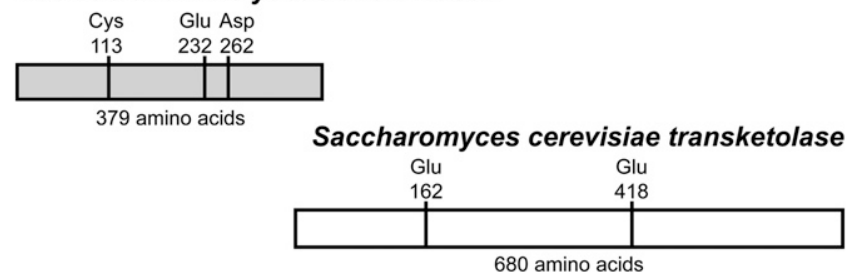

Fig. 1. The gene that encodes the thiaminase I examined in this study yields a protein with homology to both thiaminase I and transketolase enzymes. Key active-site residues are indicated.

necrosis in cattle $(9,28,29)$. Consumption of silk worm larvae as a source of protein in Nigeria causes acute seasonal cerebellar ataxia (22). Finally, the 1860-1861 expedition of Burke and Wills across Australia turned fatal when the men, whose diet consisted primarily of raw nardoo fern, suffered from thiaminase poisoning, developed beriberi, and died (30). It is tempting to speculate that these organisms have acquired thiaminase I through horizontal gene transfer and maintained it in their genomes as a mechanism of defense.

The $N$. gruberi thiaminase I is part of a large polypeptide sequence of $\sim 1,025$ aa residues (D2V4Z5; Fig. 1). The C-terminal portion of this sequence (669 aa) is homologous to transketolases. It is $49 \%$ identical in sequence to the Saccharomyces cerevisiae transketolase (P23254) and includes conservation of key residues involved in dimer stabilization, cofactor binding, and catalysis in the yeast enzyme. Attempts to isolate the full-length protein from extracts of $N$. gruberi amebae have been unsuccessful; however, the thiaminase I activity survives, indicating that the thiaminase I domain of the protein remains intact. In addition, the $\mathrm{N}$-terminal domain expressed by itself (356 aa) has thiaminase I activity in vitro that is comparable to the activity of the thiaminase I domain isolated from cellular extracts (6).

Given the low sequence identity $(\sim 25 \%)$ of the thiaminase I isolated from $N$. gruberi (Ng-thiaminase) to the previously characterized Bacillus thiaminolyticus (Paenibacillus thiaminolyticus) thiaminase I (P45741) (Bt-thiaminase) and the fact that the $N$. gruberi enzyme is part of a larger protein, we endeavored to determine its structure. Additionally, the sequence of the Btthiaminase enzyme contains only one cysteine, which is located in the active site, whereas the sequence of the $\mathrm{Ng}$-thiaminase enzyme possesses six cysteines. The structure of the Bt-thiaminase has been solved bound to a mechanism-based inhibitor (31), 4-amino-6-chloro-2,5-dimethylpyrimidine (Pyd) (Fig. S1); however, this molecule represents only the pyrimidine portion of thiamin and may not be representative of the mode of substrate binding to this class of thiaminases. Here, we describe the X-ray crystal structures of a thiaminase I from $N$. gruberi in its unliganded state and bound to a substrate analog, 3-deazathiamin (3-dzThi) (Fig. S1), which is isoelectronic with and essentially identical in shape and size to thiamin $(32,33)$. The $N$. gruberi thiaminase I has been shown to use thiamin, but not thiamin diphosphate, as a substrate and is active with both amines and sulfhydryl compounds as cosubstrates. Despite low sequence identity and differences in native polypeptide length, the tertiary structure of the thiaminase I from Naegleria gruberi closely resembles that of thiaminase I from Bacillus thiaminolyticus.

\section{Results}

Overall Structure. The structure of $\mathrm{Ng}$-thiaminase was solved using a combination of isomorphous replacement, pseudo- multiwavelength anomalous diffraction phasing, and molecular replacement (Table S1). Both the apo and holo structures of $\mathrm{Ng}$ thiaminase crystallize with six molecules in the asymmetric unit. The Ng-thiaminase has two globular domains of similar size and $\alpha / \beta$ topology, each consisting of a mixed $\beta$-sheet sandwiched by $\alpha$-helices, separated by a deep cleft at the domain interface (Fig. 2). The N-terminal domain consists of a seven-stranded $\beta$-sheet surrounded by eight $\alpha$-helices and the C-terminal domain contains a four-stranded $\beta$-sheet flanked by eight $\alpha$-helices. Numbering of the protein begins with the $\mathrm{N}$-terminal methionine of the actual protein sequence. The first four residues at the $\mathrm{N}$ terminus were not observed in the crystal structure. Amino acid residues 5-118 and 260-315 lie within the N-terminal domain, and the C-terminal domain consists of residues 119-259 and 316-356. Three peptide segments consisting of residues 118-120, 258-260, and 328-333 connect the domains. The first two segments comprise a two-stranded crossover region that forms the back of the cleft between the domains. Comparison of $\alpha$-carbon atoms using LSQMAN (34) indicates that all copies of the molecule in the asymmetric unit are essentially identical for both the apo (average rmsd, $0.13 \AA$ ) and holo (average rmsd, $0.05 \AA$ ) crystal structures.

For both the apo and holo structures of Ng-thiaminase, the contents of the asymmetric unit pack in such a way that three dimer pairs participate in a threefold screw axis where one monomer in each layer is also related by twofold symmetry to one of the monomers in the neighboring layer. This crystal packing suggests the enzyme could be a monomer or a dimer, but gel filtration experiments show that in solution $\mathrm{Ng}$-thiaminase is a monomer. The Bt-thiaminase is also a monomer.

Active Site. The assignment of the active site to a deep cleft between the $\mathrm{N}$ - and C-terminal domains is confirmed by the location of Cys118, predicted by sequence alignment with Cys113 of Bt-thiaminase to be the active site cysteine (Fig. 2 and Fig. S2). More importantly, clear electron density for one 3-dzThi molecule within this binding site for all monomers in the asymmetric unit is observed (Fig. S3). The overall character of the active site is conserved relative to the Bt-thiaminase structure (Fig. $3 B$ ). Of the 11 residues that make up the bulk of the $\mathrm{Ng}$ thiaminase active site, 8 are identical in both thiaminases (Tyr15, Tyr53, Asp68, Cys118, Tyr230, Glu232, Tyr260, Asp262) and 1 is a conservative substitution of a Phe (Phe13) for a Tyr residue. The active site of $\mathrm{Ng}$-thiaminase contains two aromatic activesite residues, Tyr-116 and Phe158, with smaller side-chain equivalents (Ile111 and Thr163) in Bt-thiaminase. There is no structural equivalent in $\mathrm{Ng}$-thiaminase for Tyr222 in Bt-thiaminase; it resides on the loop between $\alpha 10$ and $\alpha 11$, which is absent in Ng-thiaminase (Fig. S2). The predominance of aromatic residues
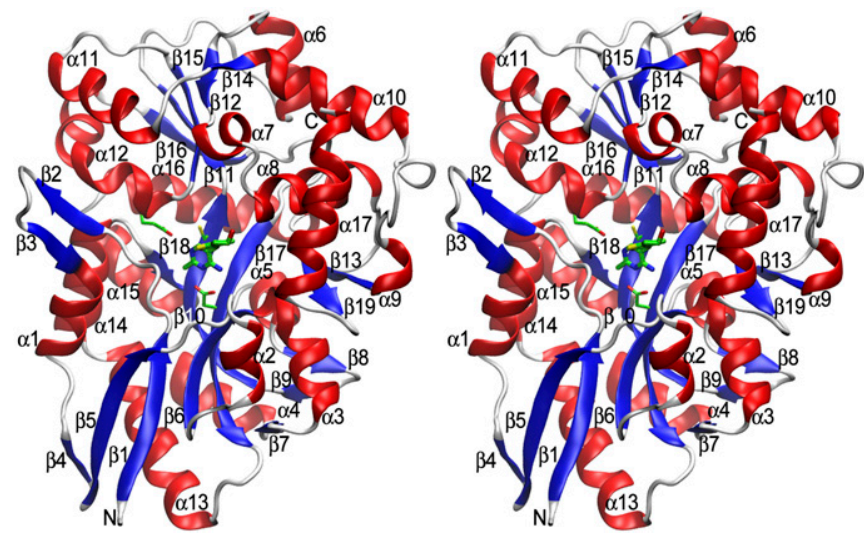

Fig. 2. Stereoview ribbon diagram of the $N$. gruberi thiaminase I bound to 3 -dzThi, a substrate analog (PDB ID code 4HCY). $\alpha$-Helices are red and $\beta$-strands are blue. 3-dzThi, Glu241, Cys118, and Asp272 are green. 
provides a mainly hydrophobic character in the active site, presumably for binding of the aromatic moieties of the substrate.

The substrate analog, 3-dzThi, bound in the Ng-thiaminase active site is a structural homolog of the substrate thiamin where $\mathrm{N} 3$ on the thiazole moiety has been substituted by C3 (Fig. S1 and Fig. 3C) $(32,33)$. Therefore, the configuration and conformation of this compound are expected to be similar to those of the substrate. 3-dzThi is bound to the Ng-thiaminase so that the pyrimidine ring is at the innermost part of the cleft that forms the active site. No covalent bond is observed between the active-site Cys118 and the inhibitor; however, the $\mathrm{C}^{\prime}{ }^{\prime}$ of the pyrimidine ring is within $3.6 \AA$ of the sulfur atom that forms a presumed covalent bond during the course of the transferase reaction. The pyrimidine ring is held in place by a large number of hydrogen bonds, possessing at least one such bond with every atom capable of making one: N1' forms hydrogen bonds to Glu232; N3' forms hydrogen bonds to Asp262; and N4' forms hydrogen bonds to Asp68 and Tyr53 and is within hydrogen-bonding distance to Asp262 (Fig. $3 A$ and $C$ ). In addition, the pyrimidine ring is surrounded by three aromatic residues (Phe13, Tyr230, and Tyr260) and is effectively clamped in place (Fig. $3 A$ ). The thiazole ring is also clamped in place by three aromatic residues (Tyr15, Tyr53, and Tyr230) and is positioned so that the plane of the ring is essentially perpendicular to the plane of the pyrimidine ring. Thus, it would seem that both rings have relatively little freedom of motion once bound in the active site and are constrained to a very specific configuration to allow the reaction to take place.

Kinetics. The Ng-thiaminase has been purified and characterized in vitro and possesses unusual stability for an enzyme. It has a half-life of 8-10 mo when stored at $0-4{ }^{\circ} \mathrm{C}$ and of 1 wk when incubated at $37^{\circ} \mathrm{C}(6)$. It is also remarkably resistant to proteolysis as the thiaminase I portion of the larger polypeptide (Fig. 1) can be isolated in its active form from whole-cell $N$. gruber lysates (6). The Ng-thiaminase uses thiamin and accepts a number of heterocyclic amines and sulfydryl compounds as the nucleophile in the cleavage reaction, including aniline, veratrylamine, $\beta$-mercaptoethanol ( $\beta$-ME), and L-cysteine (Fig. S1). By definition, the enzyme is therefore considered a thiaminase I. The kinetic parameters have been determined for aminolysis of thiamin (Table S2), with a $K_{\mathrm{M}}$ of $630 \mu \mathrm{M}$ and $k_{\text {cat }}$ of $0.16 \mathrm{~s}^{-1}$ (pH 7.0, $25^{\circ} \mathrm{C}$ ) in the presence of $40 \mathrm{mM}$ aniline and a $K_{\mathrm{M}}$ of $200 \mu \mathrm{M}$ and

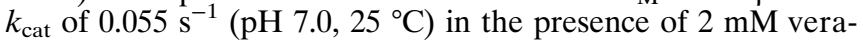
trylamine. Two nitrogenous bases were characterized as cosubstrates in the presence of $0.2 \mathrm{mM}$ thiamin (Table S2). Aniline had a $K_{\mathrm{M}}$ of $12,000 \mu \mathrm{M}$ and $k_{\text {cat }}$ of $0.091 \mathrm{~s}^{-1}$ and veratrylamine had a $K_{\mathrm{M}}$ of $180 \mu \mathrm{M}$ and $k_{\text {cat }}$ of $0.049 \mathrm{~s}^{-1}$. Two sulfhydryl compounds were also examined as potential cosubstrates. Although the enzyme can use L-cysteine, measuring the kinetic parameters for this reaction proved to be difficult under the current assay conditions even after increasing the enzyme concentration 10fold. This could be due to the inhibitory nature of sulfhydryl compounds with active-site cysteine residues through some undefined mechanism. $\beta$-ME can also act as a cosubstrate (Table $\mathrm{S} 2)$, but the reaction is relatively slow compared with the other molecules used in this study $\left(K_{\mathrm{M}}\right.$ of $3,400 \mu \mathrm{M}$ and $k_{\text {cat }}$ of 0.013 $\mathrm{s}^{-1}$ at $0.2 \mathrm{mM}$ thiamin). Bearing in mind that all standard reactions were run in the presence of $2 \mathrm{mM} \beta$-ME to keep the active-site cysteine reduced, all reactions were also run in the absence of $\beta$-ME and showed essentially no differences in $K_{\mathrm{M}}$ or $k_{\text {cat }}$ within the SE of the assay (Table S2). No product was detected from reaction mixtures containing $60 \mathrm{mM}$ aniline or $3 \mathrm{mM}$ veratrylamine, 0.1 or $0.2 \mathrm{mM}$ TPP, and greater than fivefold the amount of $\mathrm{Ng}$-thiaminase used in the standard assay conditions.

The base substitution reaction of thiamin by thiaminase $\mathrm{I}$ is proposed to proceed via a double addition-elimination mechanism based on several observations made with the enzyme from $B$. thiaminolyticus: the reaction proceeds via ping-pong kinetics $(12,13,35)$; the substitution reaction using chiral monodeuteriothiamin proceeds with retention of stereochemistry (36, 37 ); and by the mechanism-based inactivation of the enzyme with Pyd resulting in a Cys113 covalent adduct $(31,38)$. Given the similarity between Bt-thiaminase and $\mathrm{Ng}$-thiaminase, the expectation is that the mechanism should be similar if not exactly the same.

In an effort to find compounds that would show how the substrate binds, analogs of thiamin were tested for activity. Oxythiamin (Fig. S1), with which the enzyme reacts weakly in the
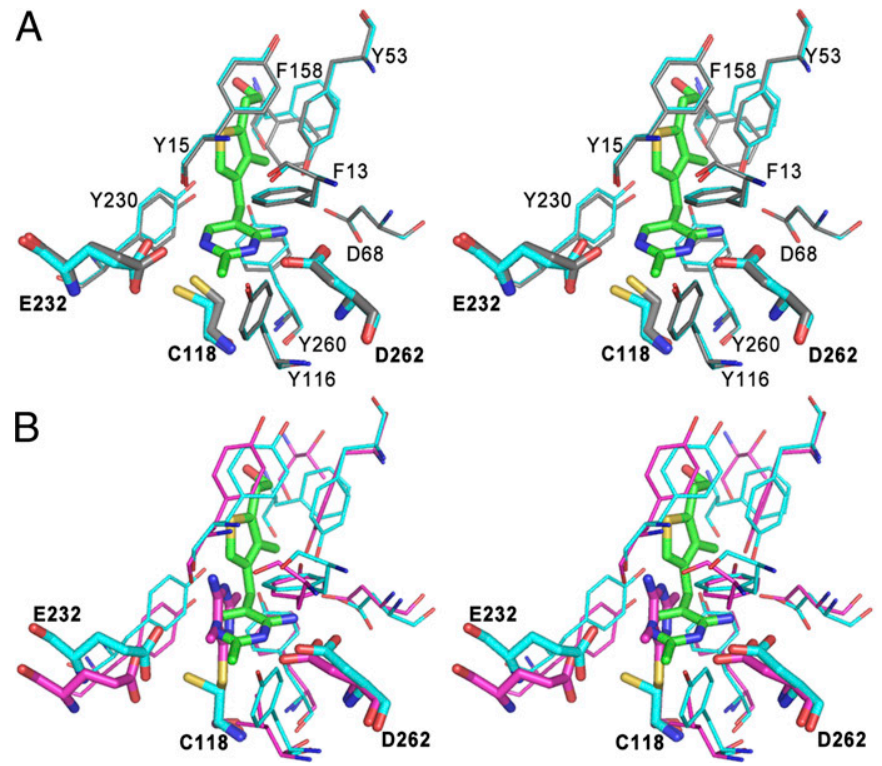
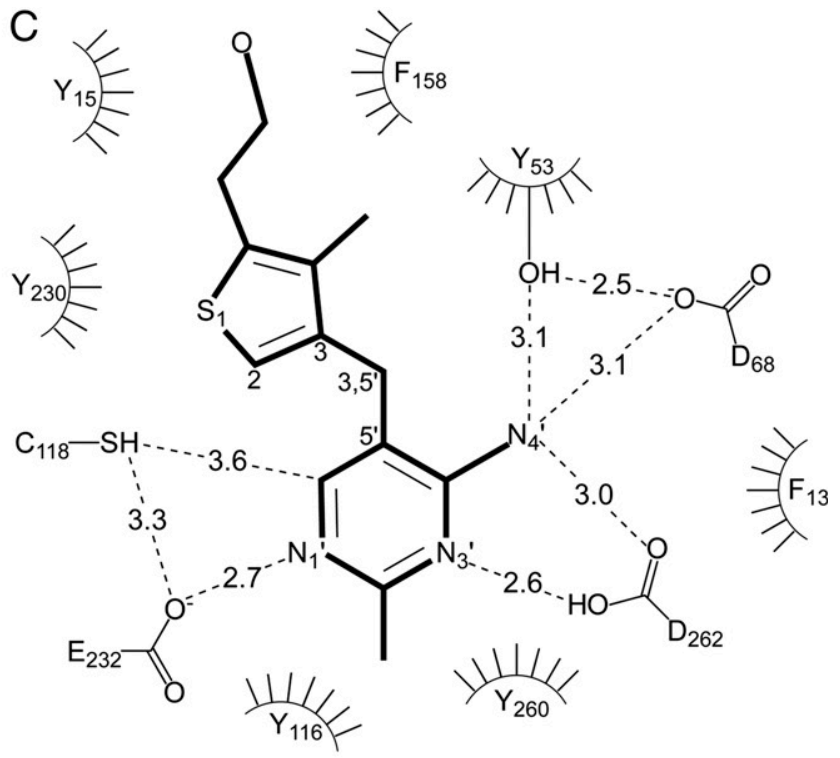

Fig. 3. The $N$. gruberi thiaminase I active site. (A) Stereoview of the superposition of apo (gray; PDB ID code 4HCW) and holo (cyan; 3 -dzThi, green; PDB code $4 \mathrm{HCY}$ ) active sites of $\mathrm{Ng}$-thiaminase. (B) Stereoview of the superposition of Ng-thiaminase (cyan; PDB ID code 4HCY) in complex with 3-dzThi (green) with the active site of Bt-thiaminase in complex with covalently bound inhibitor, Pyd [magenta; PDB ID code 4THI (31)]. For clarity, only Asp (Ng-262, Bt-272), Cys (Ng118, Bt-113), and Glu (Ng-232, Bt-241) are labeled. (C) Schematic view of the $\mathrm{Ng}$-thiaminase active site. Hydrogen bonds are represented by dashed lines, and residues involved in hydrophobic contacts to the ligand are represented by a curved comb. 
presence of $1 \mathrm{mM}$ veratrylamine, has a millimolar inhibition constant. However, 3-dzThi does not react at all, was shown to act as a competitive inhibitor with a micromolar inhibition constant, and could be used as an isosteric inhibitor to indicate the mode of binding of the substrate. Consequently, 3-dzThi was used to obtain cocrystals for structural analysis.

\section{Discussion}

Overall Structure. A search for structurally similar proteins using the DaliLite server (39) revealed, as expected, that the overall fold of $\mathrm{Ng}$-thiaminase is very similar to that of the Bt-thiaminase [Protein Data Bank (PDB) ID code 2THI] (31) and to members of the group II periplasmic-binding protein family $(40,41)$, such as the trehalose/maltose-binding protein (PDB ID code 1EU8) (42). One striking difference in the structures of these two enzymes is the insertion of an antiparallel, two-stranded " $\beta$-finger" between $\beta 1$ and $\alpha 1$ in the $\mathrm{N}$-terminal domain of the Ng-thiaminase structure that corresponds to an insertion in the N-terminal half of the Ng-thiaminase polypeptide (Fig. S2). A sequence insertion in the C-terminal half of the Bt-thiaminase sequence yields a loop of similar size, but lacking secondary structure, also in the N-terminal domain but between $\alpha 14$ and $\alpha 15$. The location of the insertion in the Ng-thiaminase sequence moves the tip of the finger closer to the opening of the active site cleft by $\sim 19 \AA$ relative to the unstructured loop observed in the Bt-thiaminase structure. The active site in the Bt-thiaminase structure is partially shielded by a loop located between $\alpha 10$ and $\alpha 11$, which is lacking both in the sequence and structure of the $\mathrm{Ng}$ thiaminase protein.

It is tempting to assign a role to the $\mathrm{Ng}$-thiaminase $\beta$-finger, and the corresponding unstructured loop in the Bt-thiaminase structure, in regulating access to the active site. However, there is no indication of a major conformational change in Ng-thiaminase upon binding of the substrate analog 3-dzThi, implying a lock-and-key mechanism over an induced-fit mechanism. The $\alpha$-carbon atoms in the apo and holo structures can be superimposed to give an rmsd of $0.23 \AA$. A similar observation can be made when comparing the apo and Pyd inhibitor-bound structures of Bt-thiaminase (rmsd, 0.51 A). For comparison, it has been demonstrated that many members of the periplasmic binding protein superfamily (40) undergo a significant hinge motion upon ligand binding. For example, the maltose binding protein undergoes a hinge motion of $35^{\circ}$ (43). Excluding the constraints of crystal packing, one possible explanation for this difference lies in the different function of the periplasmic binding proteins compared with the thiaminase I enzymes. A large hinge motion may be more advantageous to a protein that binds to, transports, and releases a ligand, than to a protein that binds a ligand and then performs catalysis.

Although gel filtration experiments indicate that the $\mathrm{Ng}$ thiaminase is a monomer in solution, the crystallized protein can be interpreted in terms of potential oligomeric structures (Fig. S4). The portion of the polypeptide sequence not included in the $\mathrm{Ng}$ thiaminase clone is homologous to transketolases, which are biologically active dimers with $\mathrm{N}$ and $\mathrm{C}$ termini arranged so that they are located on the same surface of the protein. Both of the potential $\mathrm{Ng}$-thiaminase dimers observed in the asymmetric unit are arranged so that the $\mathrm{N}$ and $\mathrm{C}$ termini of each monomer would be on the same surface of the dimer. Either potentially biologically relevant dimer could allow for the full-length protein to interact in such a way so that the transketolase domain could adopt its expected quaternary structure. There are no residues from either active site that interact with the alternate subunit in the dimer. Nevertheless, the extent of buried interface for these potential dimers is significant, and could represent dimeric interactions in the full-length protein.

Substrate Binding. The structure of Ng-thiaminase bound to the substrate analog 3-dzThi confirms the location of the active site within a cleft between the $\mathrm{N}$ - and C-terminal domains (Fig. 2) and identifies the residues involved with substrate binding (Fig.
3). The predicted active-site residue, Cys118, is located deep within this cleft and, when mutated to a serine, yields a protein that tests negative for enzymatic activity (7). Glu232 is also located in the active site and is hydrogen bonded to the substrate analog. Although one of the Glu232 side-chain carboxyl oxygen atoms interacts with $\mathrm{N}^{\prime}$ on 3 -dzThi, the other is positioned $\sim 3.3 \AA$ from the sulfhydryl group of Cys118. This glutamate is equivalent to Glu241 in the Bt-thiaminase, which, when mutated to glutamine, has been shown to be inactive (31).

In the Ng-thiaminase/3-dzThi complex and the Bt-thiaminase/ Pyd complex, the two pyrimidine rings take on very different configurations, presumably dictated by the potential interactions open to the respective compounds (Fig. 3B). For instance, a greater number of hydrogen-bonded contacts are made between the pyrimidine ring of 3-dzThi and active-site residues than are observed in the Bt-thiaminase/Pyd complex (31). The orientation of the pyrimidine moiety of 3-dzThi bound to $\mathrm{Ng}$ thiaminase is approximately perpendicular to the orientation of Pyd in complex with the Bt-thiaminase complex, with the exocyclic $\mathrm{N}^{\prime}$ nitrogens pointing in opposite directions. In the $\mathrm{Ng}$ thiaminase/3-dzThi structure, this orientation brings $\mathrm{N}^{\prime}, \mathrm{N} 3^{\prime}$, and N1' into position to form hydrogen bonds with Asp262 and Glu232. None of the analogous interactions are observed in the Bt-thiaminase/Pyd complex, but residues structurally equivalent to Tyr53, Asp68, Cys118, Tyr230, Glu232, Tyr260, and Asp262 in Ng-thiaminase are present and would be available to interact with thiamin in a similar manner. The orientation of the Pyd moiety in the Bt-thiaminase complex is presumably dictated by the covalent bond between Cys113 and $\mathrm{C}^{\prime}{ }^{\prime}$ of the inhibitor, whereas the orientation of the pyrimidine ring in the $\mathrm{Ng}$-thiaminase/ 3 -dzThi complex is dictated by the presence of the thiazole ring, which, together with the hydroxyl group, lies closer to the lip of the active site. The thiazole ring of 3-dzThi is sandwiched by a hydrophobic channel made up of Tyr15, Tyr53, Phe158, and Tyr230, and is effectively held in place. Together, these interactions orient the pyrimidine ring precisely in the active site so that Glu232 is in position to accept a proton from Cys118 when or if the sulfur makes a covalent bond to the $\mathrm{C}^{\prime}$ of the pyrimidine moiety as part of the reaction mechanism. The relevance of this orientation is supported by the previously proposed mechanism for Bt-thiaminase in which the glutamate serves as a base for activation of the active-site cysteine (31).

Unlike many enzymes that respond structurally to the presence of an inhibitor, especially one as isosteric to the substrate as this one, the active site of $\mathrm{Ng}$-thiaminase in the presence of 3-dzThi is remarkably similar to the apo structure (Fig. 3B). Although some shifts in side-chain positions are observed for Cys118 and Glu232, the largest shifts in side-chain positions are observed for Phe158, Tyr230, and Tyr260. The side chains of these aromatic residues move closer to the aromatic parts of the inhibitor, forming tighter interactions. This is in contrast to the active site of Bt-thiaminase (31), where there is a larger shift in active-site residues between the apo and inhibitor complex structures. However, it is not clear whether these differences are relevant because the Pyd inhibitor does not seem to mimic the structure of the substrate very well.

The conformation of thiamin bound to the active site of an enzyme determines whether it is a substrate or a cofactor. The conformation of thiamin is defined by the dihedral angles $\phi_{\mathrm{T}}$ $\left(\mathrm{C}^{\prime}-\mathrm{C} 3,5^{\prime}-\mathrm{N} 3-\mathrm{C} 2\right)$ and $\phi_{\mathrm{P}}\left(\mathrm{N} 3-\mathrm{C} 3,5^{\prime}-\mathrm{C}^{\prime}-\mathrm{C}^{\prime}\right)$ (44). In enzymes that use TPP as a cofactor, the higher energy "V" conformation (approximately $\phi_{\mathrm{T}}=104^{\circ}, \phi_{\mathrm{P}}=64^{\circ}$ ) is observed, whereas, in the periplasmic thiamin-binding protein, thiamin-monophosphate is bound in the low-energy " $F$ " conformation (PDB ID code 2QRY; $\left.\phi_{\mathrm{T}}=1^{\circ}, \phi_{\mathrm{P}}=-82^{\circ}\right)(45)$. The conformation of 3-dzThi is more similar to the " $\mathrm{F}$ " conformation (monomer $\mathrm{A}, \phi_{\mathrm{T}}=$ $-24.72^{\circ}, \phi_{\mathrm{P}}=-103.26^{\circ}$ ), which places the $\mathrm{N} 4^{\prime}$ amino group of the pyrimidine ring out of proximity to $\mathrm{C} 2$ on the thiazole ring. In this conformation, the $4^{\prime}$-amino group on the pyrimidine ring cannot act as a base. Additionally, the Ng-thiaminase does not 
possess the conserved sequence necessary for using TPP as a cofactor $(46,47)$.

Last, the structure of Ng-thiaminase in complex with 3-dzThi can be used as a template for the configuration of the bound substrate. Although we were unable to demonstrate utilization of TPP as a substrate by the Ng-thiaminase in this study, thiaminase I homologs from other organisms are able to use this form of thiamin $(10,23)$. Superposition of TPP onto 3-dzThi is straightforward and suggests that the pyrophosphate moiety of TPP could interact with a series of serine residues (Ser154-157) near the $\mathrm{N}$ terminus of $\alpha 8$ or possibly with Lys 346 and Tyr15. In the structure of the extracytoplasmic thiamin-binding protein, CypI (PDB ID code 3EKI), the pyrophosphate moiety of TPP is stabilized by interactions with three serine residues (Ser255257), two lysine residues (Lys129, Lys258), and Tyr215 (48). The residues necessary to make analogous interactions in the active sites of both the $N$. gruberi and $B$. thiaminolyticus thiaminase I enzymes are present.

Kinetics. Kinetic parameters have been determined for thiamin using aniline and veratrylamine as cosubstrates at $\mathrm{pH} 7.0$ and $25^{\circ} \mathrm{C}$. Compared with the B. thiaminolyticus enzyme (Table S2), the results suggest that the $N$. gruberi enzyme has both a weaker affinity for both thiamin and aniline and a slower turnover rate. These discrepancies may be due to differences in the electronic character of the different thiaminase I active sites, but could also be attributed to variations in the assay conditions, i.e., temperature and $\mathrm{pH}$. However, the results agree well with previously reported $K_{\mathrm{M}}$ values for thiamin for other eukaryotic thiaminase I enzymes including those from African silk moth $(0.35 \mathrm{mM})$, carp liver $(0.15 \mathrm{mM})$, and land snail (hepatopancreas, $0.83 \mathrm{mM}$, and headfoot, $1.06 \mathrm{mM})(17,25,26) . k_{\text {cat }}$ values for thiaminase I enzymes have not been consistently reported in the literature. Thiaminase I activity assays are typically performed with $\beta$-ME in the buffers to keep the active-site cysteine reduced; however, this molecule can also act as a cosubstrate (Table S2). In the absence of $\beta$-ME, the $N$. gruberi enzyme still turns over with comparable $k_{\text {cat }}$ values, but with somewhat lower $K_{\mathrm{M}}$ constants for the cosubstrates aniline and veratrylamine. This could be attributed to competition between thiamin and $\beta-\mathrm{ME}$ for the active-site cysteine through an undefined mechanism. Attempts were made to examine the activity of the $N$. gruberi enzyme using TPP as a substrate, but no activity was detected. TPP has been shown to react with thiaminase I from $B$. thiaminolyticus, Nardoo, and shellfish $(10,23)$.

Mechanism. The currently accepted mechanism for thiaminase I enzymes was proposed based on interactions hypothesized to take place but not observed in the structure of the Bt-thiaminase complex with a covalently bound inhibitor, Pyd (31). In the crystal structure of the $\mathrm{Ng}$-thiaminase, the arrangement of residues in the active-site cleft and the observed mode of substrate analog binding are consistent with this mechanism (Fig. $3 \mathrm{~A}$ and $C$, and Fig. S5). Cys118 is $3.6 \AA$ away from $\mathrm{C}^{\prime}$ on the pyrimidine moiety of 3 -dzThi, the proposed site of thiol addition and covalent bond formation with thiamin, which yields a carbanion intermediate and drives the elimination of the thiazole. Glu232 is positioned to serve as a base for the activation of Cys118. Addition of a nucleophile such as an amine or sulfhydryl compound on the methylene group of the pyrimidine moiety leads to elimination of the cysteine thiolate and completes the thiamin cleavage reaction. Two acidic residues, Glu232 and Asp262, interact with $\mathrm{N}^{\prime}$ and $\mathrm{N}^{\prime}$ ' of the pyrimidine moiety, respectively, but have not previously been implicated in activation of the pyrimidine.

The same mechanism has also been proposed for thiaminase II based on the structure of TenA from Bacillus subtilis bound with HMP in the active site (Fig. S6) (49). Although this enzyme has a completely different fold from that of thiaminase I, the pyrimidine of HMP makes analogous interactions with the same types of residues in TenA as does 3-dzThi with $\mathrm{Ng}$-thiaminase, including N1' hydrogen bonding to Glu205 (Glu232), N3' hydrogen bonding to Asp44 (Asp262), and N4' hydrogen bonding to Asp44 and Tyr163 (Asp262 and Tyr53) (49). Because both thiaminase I and thiaminase II catalyze the same reaction, the only difference being the type of nucleophile used, one could argue for convergent evolution between these two enzymes. Thiaminase I can use a variety of nucleophilic cosubstrates containing heterocyclic amines or sulfhydryl groups such as aniline, cysteine, DTT, pyridine, quinoline, and veratrylamine (10, 12-14), whereas thiaminase II/TenA enzymes use a hydroxyl group, exclusively water. This variety in groups transferred from another molecule to thiamin by thiaminase I enzymes may suggest different role than that of thiamin salvage in N. gruberi.

$N$. gruberi requires the addition of thiamin to its growth medium (50) and might be expected to possess the genes necessary for thiamin salvage. A preliminary search of its genome reveals that this may indeed be the case. A BLAST search using the sequences of thiamin salvage enzymes against translated nucleotide sequences in the Naegr1_scaffolds database results in hits with sequence homology to ThiE (TPS), ThiM, TPK, and ThiD as well as TenA. Two pairs of sequences with homology to ThiE/ ThiM and ThiD/TenA were found adjacent to each other on the same scaffold. It should also be noted that a second, predicted transketolase (D2VZK8) sequence with homology to the transketolase-like domain of the full-length $N$. gruberi polypeptide was found. Together, these findings make the study of this protein all of the more fascinating.

Thiaminase I Sequence Conservation. A BLAST search and COBALT multiple sequence alignment (51) of the $\mathrm{Ng}$-thiaminase sequence against sequences in the BLAST databases finds several potential homologs (Fig. S7). Analysis of the Ng-thiaminase structure and this sequence alignment using the ConSurf Server confirms the conservation of residues important to the activity of a thiaminase I enzyme (52). Residues involved in stacking interactions with the pyrimidine and thiazole moieties and residues analogous to Cys118, Glu232, and Asp262 in the Ng-thiaminase are conserved across many sequences of proteins that may possess putative thiaminase I activity. The following patterns are revealed near the following catalytic residues: Cys, G/A/S/T, $\mathrm{Y} / \mathrm{N} ; \mathrm{G}, \mathrm{F} / \mathrm{Y}, \mathrm{T} / \mathrm{S}, \mathrm{Glu}, \mathrm{X}, \mathrm{L} / \mathrm{M} ; \mathrm{F} / \mathrm{Y}, \mathrm{X}, \mathrm{Asp}, \mathrm{X}, \mathrm{L} / \mathrm{I} / \mathrm{V}$. This information, taken together with the mode of thiamin binding defined by the Ng-thiaminase/3-dzThi structure, allows for the recognition of a thiaminase signature motif.

The significance of the larger molecular weights of the eukaryotic thiaminase I enzymes remains to be determined. The Ngthiaminase structure suggests that the eukaryotic enzymes can exist as a single polypeptide, although perhaps sensitive to proteolytic modification, rather than native aggregates of smaller polypeptide subunits as observed for thiaminase II enzymes like TenA (49). It is interesting to note that Saccharomyces cerevisiae possesses multifunctional enzymes involved in thiamin salvage and biosynthesis. The C-terminal and N-terminal domains of Thi20 are homologous to TenA (thiaminase II) and ThiD (HMP/ HMP-P kinase) from $B$. subtilis, respectively (53-55). THI6 has $\mathrm{N}$ - and $\mathrm{C}$-terminal homology with ThiE (thiamin phosphate synthase) and ThiM (4-methyl-5-hydroxyethylthiazole kinase) from $B$. subtilis, respectively (56). This fusion of enzymatic activities that are key to thiamin salvage may be an emerging theme in eukaryotes. In Naegleria gruberi, the existence of a single polypeptide with both a transketolase domain, which can be predicted to use thiamin diphosphate as a cofactor, and a thiaminase I domain, which could be expected to decrease cellular levels of thiamin and thus thiamin diphosphate, seems counterintuitive. It may be the case that the thiaminase I domain provides an as-yet-to-be-determined regulatory mechanism to the transketolase domain or that the product of one domain is the substrate of the other. 


\section{Materials and Methods}

Detailed methods are given in SI Materials and Methods. Recombinant $\mathrm{Ng}$-thiaminase was expressed in and purified from an Escherichia coli expression system using an $\mathrm{N}$-terminal enterokinase cleavable His-tag. Crystallization, X-ray data collection, and subsequent structure determination were carried out on $\mathrm{Ng}$-thiaminase from which the affinity tag was removed. Thiaminase activity assays were performed using tagged protein.

ACKNOWLEDGMENTS. We are grateful to Thomas Barends and Anton Meinhart of the Max Planck Institute for Medical Research in Heidelberg; to Jacqueline Naffin-Olivos (Brandeis University); and to Mark Wilson, Raquel

1. De Jonckheere JF (2002) A century of research on the amoeboflagellate genus Naegleria. Acta Protozool 41(4):309-342.

2. Fulton $C$ (1970) Amebo-flagellates as research partners: The laboratory biology of Naegleria, Tetramitus. Methods Cell Physiol 4:341-476.

3. Fulton C (1993) Naegleria: A research partner for cell and developmental biology J Eukaryot Microbiol 40(4):520-532.

4. Fritz-Laylin LK, et al. (2010) The genome of Naegleria gruberi illuminates early eukaryotic versatility. Cell 140(5):631-642.

5. Dunnebacke TH, Dixon JS (1989) NACM, a cytopathogen from Naegleria ameba: Purification, production of monoclonal antibody, and immunoreactive material in NACM-treated vertebrate cell cultures. J Cell Sci 93(3):391-401.

6. Fulton C, Lai EY (2010) Methods for inducing apoptosis by reducing the level of thiamin. US Patent 7,803,531 B2.

7. Fulton C, Lai EY (2010) Thiaminases and thiaminase genes for use in apoptotic therapies. US Patent 7,736,898 B1.

8. Carpenter KJ (2000) Beriberi, White Rice and Vitamin B: A Disease, a Cause, and a Cure (Univ of California, Berkeley, CA).

9. Evans WC (1975) Thiaminases and their effects on animals. Vitam Horm 33:467-504.

10. Murata K (1982) Actions of two types of thiaminase on thiamin and its analogues. Ann N Y Acad Sci 378:146-156.

11. Wittliff JL, Airth RL (1970) Thiaminase II (thiamine hydrolase, EC 3.5.99.2). Methods Enzymol 18A:234-238.

12. Lienhard GE (1970) Kinetic evidence for a (4-amino-2-methyl-5-pyrimidinyl)methylenzyme intermediate in the thiaminase I reaction. Biochemistry 9(15):3011-3020.

13. Costello CA, Kelleher NL, Abe M, McLafferty FW, Begley TP (1996) Mechanistic studies on thiaminase I. Overexpression and identification of the active site nucleophile. J Biol Chem 271(7):3445-3452.

14. Fujita A (1955) Thiaminase. Methods Enzymol 2:622-628.

15. Wittliff JL, Airth RL (1968) The extracellular thiaminase I of Bacillus thiaminolyticus. I. Purification and physicochemical properties. Biochemistry 7(2):736-744.

16. Agee CC, Wilkins JH, Airth RL (1973) Cell-bound thiaminase I of Bacillus thiaminolyticus. J Bacteriol 115(3):949-956.

17. Boś M, Kozik A (2000) Some molecular and enzymatic properties of a homogeneous preparation of thiaminase I purified from carp liver. J Protein Chem 19(2):75-84.

18. Fujita A (1954) Thiaminase. Adv Enzymol Relat Subj Biochem 15:389-421.

19. Fujita A (1972) Recollections of the background of the study on thiaminase and thermostable thiamine-decomposing factor in Japan. J Vitaminol (Kyoto) 18(1):67-72.

20. Hayashi R, et al. (1964) Studies on thiaminase of the Clostridium. I. Thiaminase of Clostridium sporogenes. J Vitaminol (Kyoto) 10(2):168-171.

21. Nishimune T, Watanabe $Y$, Okazaki H (2008) Studies on the polymorphism of thiaminase I in seawater fish. J Nutr Sci Vitaminol (Tokyo) 54(5):339-346.

22. Nishimune T, Watanabe $Y$, Okazaki H, Akai H (2000) Thiamin is decomposed due to Anaphe spp. entomophagy in seasonal ataxia patients in Nigeria. J Nutr 130(6): $1625-1628$.

23. McCleary BV, Chick BF (1977) The purification and properties of a thiaminase I enzyme from nardoo (Marsilea drummondii). Phytochemistry 16(2):207-213.

24. Wittliff JL, Airth RL (1970) Thiaminase I (thiamine: base 2-methyl-4-aminopyrimidine5-methenyltransferase, EC 2.5.1.2). Methods Enzymol 18A:229-234.

25. Okonji RE, Bamitale KS, Balogun RO (2012) Biochemical properties of thiaminase from Anaphe venata Burtler. Afr J Biotechnol 11(20):4543-4550.

26. KuKu A, Okonji RE, Akinrinola BD (2012) Comparative study of thiaminase in headfoot and hepatopancreas of Limicolaria flammea (Müller, 1774). Int J Biochem Res Rev 2(1):31-49.

27. Jurgenson CT, Begley TP, Ealick SE (2009) The structural and biochemical foundations of thiamin biosynthesis. Annu Rev Biochem 78:569-603.

28. Honeyfield DC, Brown SB, Fitzsimons JD, Tillitt D (2005) Early mortality syndrome in Great Lakes salmonines. J Aquat Anim Health 17(1):1-3.

29. Ramos JJ, et al. (2003) Biochemical changes in apparently normal sheep from flocks affected by polioencephalomalacia. Vet Res Commun 27(2):111-124.

30. Earl JW, McCleary BV (1994) Mystery of the poisoned expedition. Nature 368(6473): 683-684.

31. Campobasso N, Costello CA, Kinsland C, Begley TP, Ealick SE (1998) Crystal structure of thiaminase-I from Bacillus thiaminolyticus at $2.0 \AA$ A resolution. Biochemistry 37(45): 15981-15989.

32. Hawksley D, Griffin DA, Leeper FJ (2001) Synthesis of 3-deazathimine. J Chem Soc Perkin Trans 1(2):144-148.
Lieberman, Dali Liu, and Quyen Hoang (formerly of Brandeis University) for helpful discussions. Use of the Advanced Photon Source was supported by the US Department of Energy (DOE), Basic Energy Sciences, Office of Science, under Contract DE-AC02-06CH11357. Use of BioCARS-CAT Sector 14 was supported by the National Institutes of Health, National Center for Research Resources. Use of GM/CA-CAT Sector 23 was supported in whole or in part with federal funds from the National Cancer Institute (Y1-CO-1020) and the National Institute of General Medical Science (Y1-GM-1104). Portions of this research were carried out at the Stanford Synchrotron Radiation Lightsource, a national user facility operated by Stanford University on behalf of the DOE, Office of Basic Energy Sciences. The Stanford Synchrotron Radiation Lightsource (SSRL) Structural Molecular Biology Program is supported by the DOE Office of Biological and Environmental Research, and by the National Institutes of Health, National Center for Research Resources, and the National Institute of General Medical Sciences.

33. Mann S, Perez Melero C, Hawksley D, Leeper FJ (2004) Inhibition of thiamin diphosphate dependent enzymes by 3-deazathiamin diphosphate. Org Biomol Chem 2(12):1732-1741.

34. Kleywegt GJ, Jones TA (1994) Halloween... masks and bones. From First Map to Final Model, eds Bailey S, Hubbard R, Waller D (SERC Daresbury Laboratory, Warrington, UK), pp 59-66.

35. Puzach S, Gorbach Z, Ostrovskii Y (1984) Isolation, purification, and certain properties of thiaminase I from Anodontacygnea. Biochem Mosco 49(7):1010-1016.

36. Nicewonger R, Rammelsberg A, Costello CA, Begley TP (1995) Mechanistic studies on thiaminase I: 1. The stereochemical course of the reaction. Bioorg Chem 23(4):512-518.

37. Nicewonger R, Costello CA, Begley TP (1996) Mechanistic studies on thiaminase I. 3 . Stereochemistry of the thiaminase $\mathrm{i}$ and the bisulfite-catalyzed degradation of chiral monodeuteriothiamin. J Org Chem 61(12):4172-4174.

38. Hutter JA, Slama JT (1987) Inhibition of thiaminase I from Bacillus thiaminolyticus. Evidence supporting a covalent 1,6-dihydropyrimidinyl-enzyme intermediate. Biochemistry 26(7):1969-1973.

39. Hasegawa H, Holm L (2009) Advances and pitfalls of protein structural alignment. Curr Opin Struct Biol 19(3):341-348.

40. Quiocho FA, Ledvina PS (1996) Atomic structure and specificity of bacterial periplasmic receptors for active transport and chemotaxis: Variation of common themes. Mol Microbiol 20(1):17-25.

41. Fukami-Kobayashi K, Tateno Y, Nishikawa K (1999) Domain dislocation: A change of core structure in periplasmic binding proteins in their evolutionary history. $\mathrm{J} \mathrm{Mol} \mathrm{Biol}$ 286(1):279-290.

42. Diez J, et al. (2001) The crystal structure of a liganded trehalose/maltose-binding protein from the hyperthermophilic Archaeon Thermococcus litoralis at $1.85 \AA$. J Mol Biol 305(4):905-915

43. Sharff AJ, Rodseth LE, Spurlino JC, Quiocho FA (1992) Crystallographic evidence of a large ligand-induced hinge-twist motion between the two domains of the maltodextrin binding protein involved in active transport and chemotaxis. Biochemistry 31(44):10657-10663

44. Pletcher J, Sax M, Turano A, Chang C (1982) Effects of structural variations in thiamin, its derivatives and analogues. Ann N Y Acad Sci 378(1):454-458.

45. Soriano EV, et al. (2008) Structural similarities between thiamin-binding protein and thiaminase-I suggest a common ancestor. Biochemistry 47(5):1346-1357.

46. König S, Schellenberger A, Neef H, Schneider G (1994) Specificity of coenzyme binding in thiamin diphosphate-dependent enzymes. Crystal structures of yeast transketolase in complex with analogs of thiamin diphosphate. J Biol Chem 269(14): 10879-10882.

47. Leeper FJ, Hawksley D, Mann S, Perez Melero C, Wood MD (2005) Studies on thiamine diphosphate-dependent enzymes. Biochem Soc Trans 33(Pt 4):772-775.

48. Sippel $\mathrm{KH}$, et al. (2009) Structural insights into the extracytoplasmic thiamine-binding lipoprotein p37 of Mycoplasma hyorhinis. J Bacteriol 191(8):2585-2592.

49. Toms AV, Haas AL, Park JH, Begley TP, Ealick SE (2005) Structural characterization of the regulatory proteins TenA and Tenl from Bacillus subtilis and identification of TenA as a thiaminase II. Biochemistry 44(7):2319-2329.

50. Fulton C, Webster C, Wu JS (1984) Chemically defined media for cultivation of Naegleria gruberi. Proc Natl Acad Sci USA 81(8):2406-2410.

51. Papadopoulos JS, Agarwala R (2007) COBALT: Constraint-based alignment tool for multiple protein sequences. Bioinformatics 23(9):1073-1079.

52. Ashkenazy H, Erez E, Martz E, Pupko T, Ben-Tal N (2010) ConSurf 2010: Calculating evolutionary conservation in sequence and structure of proteins and nucleic acids. Nucleic Acids Res 38(Web Server issue):W529-W533.

53. Haas AL, Laun NP, Begley TP (2005) Thi20, a remarkable enzyme from Saccharomyces cerevisiae with dual thiamin biosynthetic and degradation activities. Bioorg Chem 33(4):338-344.

54. Onozuka M, Konno H, Kawasaki Y, Akaji K, Nosaka K (2008) Involvement of thiaminase II encoded by the THI20 gene in thiamin salvage of Saccharomyces cerevisiae. FEMS Yeast Res 8(2):266-275.

55. French JB, Begley TP, Ealick SE (2011) Structure of trifunctional THI20 from yeast. Acta Crystallogr D Biol Crystallogr 67(Pt 9):784-791.

56. Paul D, Chatterjee A, Begley TP, Ealick SE (2010) Domain organization in Candida glabrata THI6, a bifunctional enzyme required for thiamin biosynthesis in eukaryotes. Biochemistry 49(45):9922-9934. 